\title{
A RAPID HYDROGEN ELECTRODE METHOD FOR DETERMINATION OF HYDROGEN ION CONCEN- TRATIONS IN BACTERIAL CULTURES OR IN OTHER TURBID OR COLORED SOLUTIONS
}

\author{
HARRY M. JONES \\ From the Department of Pathology and Bacteriology, University of Illinois, \\ College of Medicine, Chicago
}

Recently the determination of hydrogen ion concentrations has come into such general use in biologic work that the question often arises as to the choice of a method for this purpose.

The colorimetric method has proven suitable for most routine bacteriologic work, and has the advantage of yielding a large number of determinations in a given period of time. Its limitations however are noteworthy. In general, it does not permit of more than a fair degree of accuracy especially for colored or turbid solutions ; for opaque solutions it cannot be used; when large percentages of salt or protein are in solution, its results are not dependable because of the so-called "salt" and "protein errors" of indicators; and the preparation of the standard solutions, in which one is required to assemble a great number of calibrated burets, pipets, bottles, etc., and to recrystalize, dry to constant weight and accurately weigh a number of reagents, many of which are difficult to obtain, makes it a cumbersome method.

On the other hand, the extreme accuracy possible by the hydrogen electrode method, with potentiometer and constant temperature device, is seldom required in biologic investigation. Moreover, the cost of such equipment is prohibitive for many laboratories.

More simplified outfits are described in texts on physical chemistry, but the obvious objection to them is the relatively enormous amount of time consumed in saturating the solution with hydrogen gas. This difficulty is obviated by observing the principle suggested by Hasselbach ${ }^{1}$ in which the solution and gas are brought into equilibrium in a few minutes by shaking in a closed vessel.

The vessel here described possesses no advantages over those described by $\mathrm{Clark}^{2}$ and by $\mathrm{McClendon}$ and others except that it is easily constructed, even by one of only average technical skill, and from such material as is

Received for publication May 1, 1919.

1 Biochem. Ztschr., 1911, 30, p. 317. 
commonly found in any biologic laboratory. In urging the adoption of this nethod, simplicity of apparatus and technic should be its recommendations; but it seems that the method has been too much avoided by biologists, who, though recognizing its value, have regarded it as one belonging exclusively :o the physical chemist. Nothing is perhaps more to blame for this, however, than the multiplcity of symbols, formulas, measurements, etc., which only confuse rather than enlighten the uninitiated. An attempt at simplifying the relations of the measurements to the mathematics of the method is therefore justified.

\section{The Meaning of $\mathrm{Ph}_{\mathrm{H}}$}

The concentration of any acid in a given solution is defined on the basis of its normality, namely, $1 \mathrm{gm}$. equivalent of hydrogen (not hydrogen ion) per liter. The concentration of ionic hydrogen is defined on the same basis, namely, $1 \mathrm{gm}$. equivalent of hydrogen ion (not hydrogen) in one liter and is called normal hydrogen ion. The concentration of hydrogen ion in neutral water for example is $\frac{\mathrm{N}}{10,000,000}$. The reason for the unwieldiness of this expression is evident and a more convenient method of referring to these extreme dilutions of hydrogen ion near the neutral point is demanded. Therefore, using the $N / 1$ solution as the basis of comparison, by referring to the number of times which a given solution is tenfold more dilute than $N / 1$, the expression indicating its concentration is much shortened, and yet quite as significant for purposes of comparing with other concentration. This number Sörensen called $\mathrm{P}_{\mathrm{H}}$.

In accordance with this, then, the $P_{H}$ of an $N / 100$ hydrogen ion solution is 2, namely, 2 tenfold dilutions of a $\mathrm{N} / 1$ solution; of $\mathrm{N} / 10,000$ it is 4 ; $\mathrm{N} / 10,000,000$ it is 7 , etc.

Obviously, the $\mathrm{P}_{\mathrm{H}}$ of a solution is simply the logarithm of its dilution, namely, $\log \mathrm{V} .^{3}$

Measuring the $\mathrm{P}_{\mathrm{H}}$ of a Solution: With due consideration for saturation with hydrogen gas and other appropriate steps in the technic of a determination, uniting two solutions of $\mathrm{N} / 1$ and $\mathrm{N} / 10$ through a U-tube containing strong $\mathrm{KCl}$, and then joining the platinum electrodes, immersed in the solutions to the terminals of a voltmeter, one may prove that the platinum electrode in the more dilute solution is 58 millivolts less positive than the other. By keeping the dilution in one vessel at $\mathrm{N} / 1$ (normal hydrogen electrode) and increasing the dilution in the other by tenfold each time, it will be seen that for each tenfold increase in the dilution in this vessel, an additional increase of 58 millivolts is registered by the voltmeter. Therefore, if the $P_{H}$ of any given solution is unknown, the voltage developed by it, divided by 58 millivolts, namely, $\frac{E . M . F}{58}$, gives the number of its tenfold dilutions, namely,
its $\mathrm{P}_{\mathrm{H}}$.

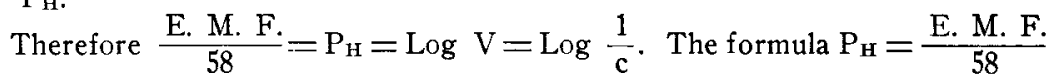
is used provided one of the solutions is kept at N/1 (normal hydrogen electrode) as indicated. In practice, however, the normal calomel electrode

2 Jour. Biol. Chem., 1915, 23, p. 475.

8 Since dilution (V) is the reverse of $(C)$, i. e., concentration, the reverse of $(C)$, i. e., $\left(\frac{1}{C}\right)$ is commonly used in place of (V) whence the expression Log $\frac{1}{C}$. But the objection to the expression $\log \frac{1}{C}$ is that it is easier to think of the logarithm of a whole number, $\log V$, than of the logarithm of the reciprocal of a fraction. 
instead of the normal hydrogen electrode is used, and since it is 280 millivolts more positive than the normal hydrogen electrode, 280 must be subtracted from the E. M. F. The equation then becomes $P_{H}=\frac{E . M . F .-280}{58}$. The element of temperature also enters into the equation in very accurate work, but between the temperature of 20 and $30 \mathrm{C}$., no correction will be necessary for readings accurate to 1 millivolt. The relation of temperature to the other factors is expressed in the following equations $P_{H}=\frac{E . M . F-0.28}{0.000198 \times T}$, in which
$T=$ the absolute temperature.

\section{The Hydrogen Electrode}

Clark ${ }^{4}$ maintains that the old method of bubbling hydrogen gas through a culture whose hydrogen ion concentration is to be determined is useless. From four to twenty-four hours are necessary to attain saturation with the hydrogen, and consequently important changes in reaction by this time have occurred. Hasselbach," however, showed that if the culture fluid is confined in a vessel filled with $\mathrm{H}$ gas, saturation is very quickly attained by shaking the solution and gas together.

Then Acree and Myers showed that if complete saturation is to be obtained the containing vessel must have no dead spaces in its interior, namely, entrance or exit tubes, where portions of the fluid or gas can hide and thus prevent the remainder of the contents from continuing at equilibrium after the shaking process is stopped.

It is known that when two dissimilar solutions are brought into contact with each other, a small E. M. F. is generated at the zone of contact. Since this E. M. F. would cause a slight error* in the reading of the voltage developed at the electrodes themselves, the solution in the hydrogen electrode vessel after saturation with hydrogen gas is, in practice, brought into contact with the solution in the calomel electrode by means of a connecting column of $\mathrm{KCl}$ (about $3 \mathrm{~N}$ ). Cummings and Gilchrist have shown that this contact should be made in such a way that the solution saturated with hydrogen lies on the connecting solution of $\mathrm{KCl}$ in a zone whose area is not too small.

The electrode vessel here described was designed with these various suggestions in mind. Its parts are assembled as follows: A test tube of about 1 inch diameter is cut off at both ends, making a cylinder of about 3 inches long of the middle portion. Both ends should be burnished, and then properly fitted with 2-hole rubber stoppers. One of these stoppers is fitted with two L-tubes bent as illustrated, and sealed at their inner ends. A pinhole is made at each of these inner ends about $1 / 8$ inch from the tip. This is most easily done while the ends are still hot following the sealing process, by boring a red hot platinum needle through the wall of the glass tube, withdrawing, and then, by means of a file, breaking off the thread of glass which follows the needle, and filing the surface around the pinhole until smooth. One of these

* To measure the extent of this error, one may show by the hydrogen electrode apparatus that the chain $\mathrm{H}_{2}|\mathrm{~N} / 10 \mathrm{H} \mathrm{Cl}| \mathrm{K} \mathrm{Cl}|\mathrm{N} / 100 \mathrm{H} \mathrm{Cl}| \mathrm{H}_{2}$, develops an $\mathbf{E}$. $\mathbf{M}$. F. of 0.058 volts (since one solution has a tenfold greater concentration of hydrogen ion that the other); whereas, the chain $\mathbf{H}_{2}|\mathrm{~N} / 10 \mathrm{HCl}| \mathrm{N} / 100 \mathrm{HCl} \mid \mathrm{H}_{2}$ develops only 0.019 volt. In other words, the $E$. M. F. developed at the zone of contact between the $N / 10$ and $N / 100$ solutions is 0.058-0.019 or 0.039 volts, and acts in opposition to the $E$. M. F. developed by the two electrodes, thereby reducing their voltage from 0.058 volts to 0.019 volts. This error of electrodes, thereby reducing their voltage from 0.058 volts to 0.019 volts, This error of
0.039 volts, or $39 \mathrm{mv} .$, is obviously too large to ignore in practice, and is due, as stated above, to the potential of the contact zone opposing the potential of the two electrodes.

- Jour. Biol. Chem., 1915, 23, p. 475.

- Biochem. Ztschr., 1911, 30, p. 317.

- Am. Chem. Jour., 1913, 50, p. 396.

7 Tr. Faraday Soc., 1913, 19, p. 174. 
Rapid Determination of $\mathrm{P}_{\mathrm{H}}$

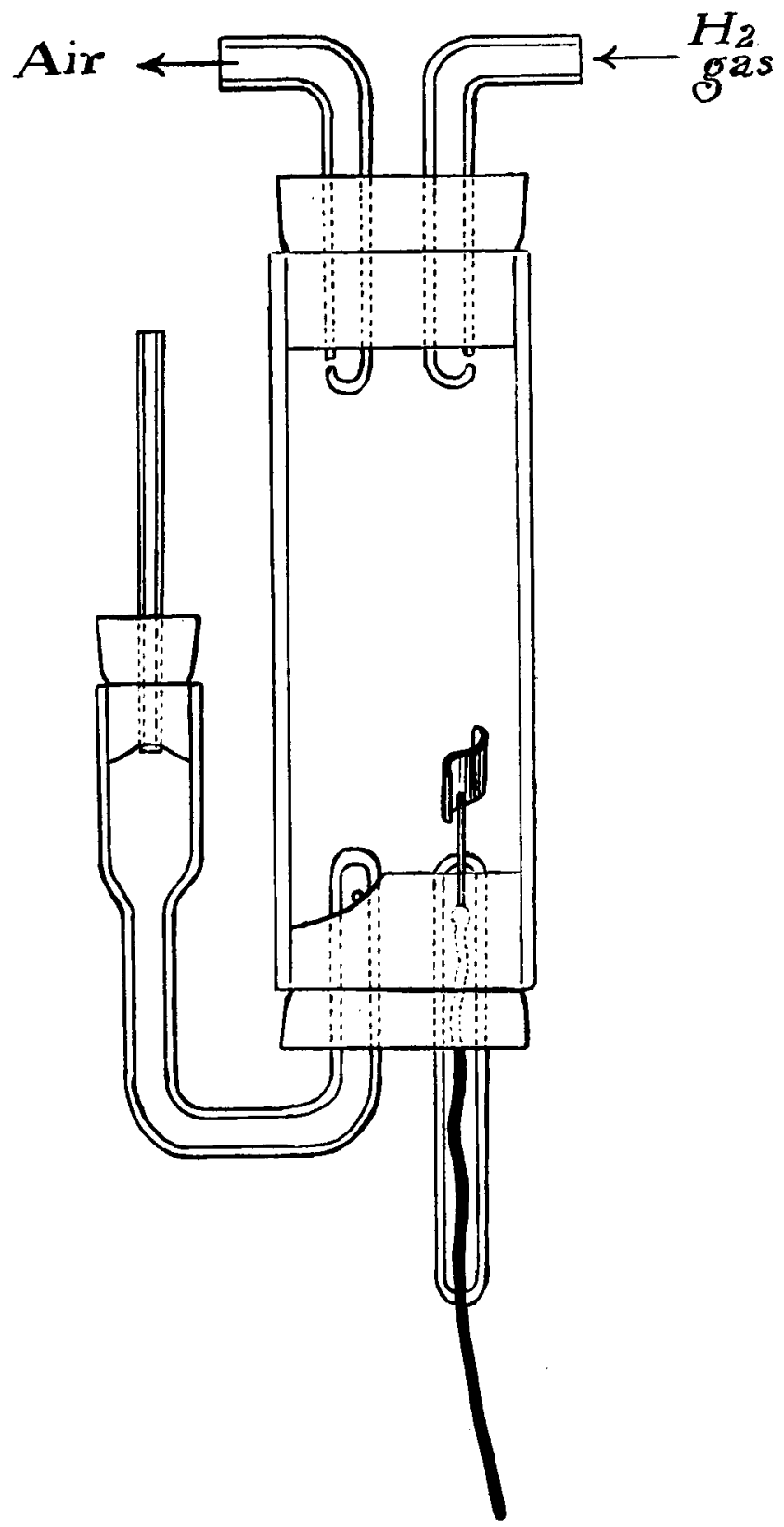

Fig. 1.-Diagram of hydrogen electrode. 
pinholes, when the tubes are pushed in beyond the body of the stopper, serves as an entrance for hydrogen, the other as an exit for the air which is to be displaced.

An oblique slice is cut from the inner end of the other stopper as illustrated. This hole is fitted with the J-tube which will be used to contain the $3 \mathrm{~N} \mathrm{KCl}$ solution (for connecting the solution saturated with $\mathrm{H}$ gas with the solution in the calomel electrode). The other hole receives the glass tube through which passes the copper wire to the platinum electrode. The J tube also has a pinhole near its tip, as illustrated, which should be rather large.

The platinum electrode is made by heating a bit of platinum plate and platinum wire to white heat on some smooth surface and then welding them together with a hot hammer. The platinum wire is then fused to the copper wire by holding the latter with some nonconducting substance, as cork, and plunging into a blow pipe or good bunsen flame until its tip softens to a small drop of molten copper, and then bringing the free tip of the platinum wire into the flame until it just touches the molten copper. The operation must be done quickly else a large ball of molten copper forms on the end of the wire. The copper wire is then placed within a short piece of glass tubing and the end of the latter sealed about the platinum wire portion as illustrated.

The large end of the $J$ tube is to be closed by a small rubber stopper the smaller end of which is cut as illustrated, and through which passes a piece of open capillary glass tubing. The electrode vessel is then complete.

\section{The Determination}

The purpose of the operation, when using the type of electrode vessel here described, is to fill the J-tube with the $3 \mathrm{~N} \mathrm{KCl}$ connecting solution, place in the vessel some of the solution to be tested, displace the air with $\mathrm{H}_{2}$ gas, shake until the solution is saturated, make electrolytic contact between the latter and the calomel electrode, measure the E. M. F. developed, and from this, calculate the $P_{H}$ of the solution.

The details of these various steps follow: Remove the small stopper with open capillary tubing from the end of the J-tube. Turn the J-tube so that the pin-hole near the tip of its other end appears. Remove the rubber stopper with the two $L$ tubes from the vessel, and with a pipet run in to the $J$-tube some strong $\mathrm{KCl}$ solution (about $3 \mathrm{~N}$ ) until all the air is driven out by way of the pin-hole. Now turn the J-tube back again through about 90 degrees so that the pin-hole disappears. The $\mathrm{KCl}$ solution is thus held in the J-tube by the pressure of the rubber stopper over the pin-hole. Continue to run in $\mathrm{KCl}$ solution into the J-tube up to within $1 \mathrm{~cm}$. of the top. Replace the small rubber stopper in such a way that the air bubble and excess of $\mathrm{KCl}$ solution will escape through the open capillary tube.

Wash out the vessel with some of the solution to be tested, then use enough of the latter to just cover the top edge of the platinum electrode. Replace the rubber stopper, having previously pushed in the L-tubes until their pin-holes are just visible. Now run in a slow stream of pure hydrogen gas by way of the L-tubes, for about 30 seconds, or until the air is all driven out by way of the other L-tube. Shut off the flow of hydrogen gas, gently withdraw the air exit L-tube just far enough to effectually close the pin-hole by the pressure of the rubber stopper, then in a similar way withdraw the other L-tube. We now have the solution to be tested in an atmosphere of hydrogen gas, and enclosed in a space which has no side pockets or "dead" spaces into which fluid or gas can hide and thus fail to reach equilibrium with the rest of the vessel contents. 
After shaking the vessel for about one minute the small rubber stopper and capillary tube are withdrawn, and the J-tube again turned back far enough for the pin-hole to be seen. If this is done slowly, the $\mathrm{KCl}$ solution will be seen to flow in and quietly settle in a layer in the slight depression where the oblique slice of the rubber stopper was cut away. This then affords a wide zone of contact bettween the $\mathrm{KCl}$ solution and the solution to be tested. The vessel is now ready to be connected to the calomel electrode. This is done by simply adjusting the latter so that the tip of its connecting tube is dipped into the $\mathrm{KCl}$ solution in the J-tube.

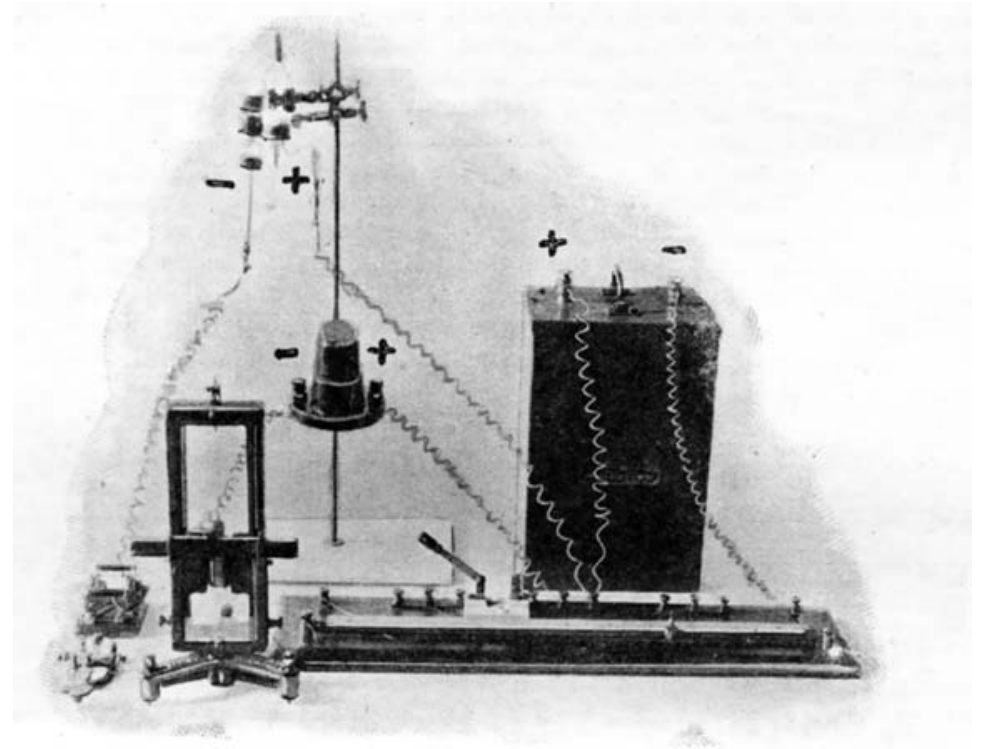

Fig. 2.-Photograph of apparatus for making hydrogen ion concentration determinations: Wheatstone bridge, Harvard switch, telegraph key, galvanometer, Weston standard cell, 2-volt lead accumulator, normal calomel electrode and hydrogen electrode. The positive and negative elements are indicated, and their connections easily traced. The bridge is shortened by laying on the $50 \mathrm{~cm}$. scale only that section of the bridge wire commonly used in measurements made on biologic fluids, namely, the sections falling between 400 and 900 $\mathrm{mm}$. from the positive end of the bridge.

It is desirable, if not necessary, to test the uniformity of the platinum electrodes after these have been platinized. For this, the author uses a vessel like the one already described, but large enough so that the rubber stopper has holes for three or four platinum electrodes instead of one. Once the platinum black on each electrode has become saturated with hydrogen gas the voltage developed by each should be identical. Differences are readily detected if present, and will probably be due to unclean platinum wire or plate. When properly platinized, the color of the electrode is a rich velvet black, but with use changes to brown, when it should be replatinized. The first determination made with a freshly platinized electrode will require several minutes more than subsequent ones for the reason that more time is required for the platinum black to become saturated with the hydrogen gas. 
From a preceding paragraph it is seen that as the ionic dilution of a given solution is increased ten times, say from $N / 1$ to $N / 10$, its $P_{H}$ is increased by 1 , and its voltage by 0.058 volts. Therefore 0.058 volts or 58 millivolts $=1 \mathrm{P}_{\mathrm{H}}$. Then $1 \mathrm{mv}=0.02 \mathrm{PH}_{\mathrm{H}}$ (about). An error, then, of $1 \mathrm{~mm}$. in our reading would mean an error of 0.02 of a $P_{\mathrm{H}}$. The apparatus is easily accurate to $0.5 \mathrm{mv}$. or $0.01 \mathrm{P}_{\mathbf{H}}$. The author has found it desirable to combine the principles of the colorimetric method with the gas chain method, in those instances where large numbers of determinations are to be made as in bacterial cultures. This is done by taking a portion $(2 \mathrm{cc})$ of each solution to be tested, diluting with $4 \mathrm{cc}$ of distilled water, and after arranging the tubes in about three groups with reference to their degree of turbidity, placing each group in separate rows in test tube racks. Equal amounts of some indicator, with a range of color change adapted to the probably "acidities" of the solutions (one of those devised and recommended by Clark and $\mathrm{Lubs}^{8}$ ) is placed in each tube and shaken. In each group the colors developed enable one at once to collect the tubes into subgroups of similar colors. One specimen of each subgroup when tested with the hydrogen electrode, then gives the $\mathrm{H}$-ion concentration of the entire subgroup, thus obviating the necessity of preparing standard solutions for color comparisons. In one series of determinations on about 80 cultures, it was found necessary when using this method to make actual measurements on only 6 specimens of the entire lot.

\section{SUM MARY}

A new apparatus for determining the dilution of hydrogen ion in bacterial cultures and other fluids is described. As compared with the colorimetric method, the apparatus here described has wider application, is more accurate, less cumbersome, and only slightly less rapid.

The hydrogen electrode vessel described, was designed with two objects, chiefly, in mind: (1) to provide a vessel accurate at least to $0.01 \mathrm{P}_{\mathbf{H}}$ and (2) to provide a vessel giving rapid saturation with hydrogen gas, and yet one which is easily constructed.

A rapid and labor saving technic combining the indicator, and the gas-chain methods is described, which obviates the difficult task of preparing standard solution for the former method, and of making needless repetitions by the latter.

\footnotetext{
8 Jour. Infect. Dis., 1915,17 , p. 160.
} 